\title{
Infrared thermographic detection and characterisation of impact damage in carbon fibre composites: results of the round robin test
}

\author{
by V. P. Vavilov ${ }^{1}$, D. P. Almond ${ }^{2}$, G. Busse ${ }^{3}$, E. Grinzato ${ }^{4}$, J.-C. Krapez ${ }^{5}$, \\ X. Maldague ${ }^{6}$, S. Marinetti ${ }^{4}$, W. Peng ${ }^{2}$, V. Shirayev ${ }^{1}$ and D. $\mathrm{Wu}^{3}$
}

'Tomsk Polytechnic University, Russia, 634028, Tomsk, 28, Savinykh St., 3

${ }_{3}^{2}$ School of Materials Science, University of Bath, Bath, BA2 7AY, U.K.

${ }^{3}$ Institut für Kunstoffprüfung und Kunststoffkunde, Universität Stuitgart, Pfaffenwaldring 32, D-70569 Stuttgart, Germany

${ }_{5}^{4}$ ITEF-CNR, Corso Stati Uniti, 4, 35127-Padua, Italy

${ }^{5}$ Damage and Solid Mechanics Department, ONERA, BP 72, 92322 Chatillon cedex, France

${ }^{6}$ Université Laval, Cité Universitaire, Québec, Canada, G1K 7P4

\section{Abstract}

Results of inspecting an impact damaged carbon fibre composite specimen are reported. Several physical test procedures and processing algorithms have been analysed within the framework of the international Round Robin Test. A stafistical means for rating non-destructive testing techniques is proposed to convert original infrared images to defect maps that are of special interest for end-users. The potentials of the techniques for characterising shape and depth distribution of impact damage in carbon fibre composites are discussed, including comparison with ultrasonic C-scan results.

\section{Introduction}

In dynamic thermal non-destructive testing (TNDT), a number of novel data processing techniques have recently become available: modulated (thermal wave) thermography, timeresolved radiometry, dynamic thermal tomography, early detection method, IR image treatment in the Fourier domain, etc. [1-5]. The TNDT procedures under discussion involve a Diracppulse (flash), square pulse (or quasi continuous pulse) and modulated heating.

In 1996, the EUROTHERM Working Group on Quantitative Infrared Thermography (QIRT) agreed to launch a Round Robin Test (RRT) to analyse the peculiarities of available TNDT techniques when applied to the same reference specimen.

In this paper, general results obtained from a carbon fibre composite specimen with the impact damage are presented.

\section{Principles of data evaluation}

\subsection{Statistical critenia}

In this study, we have concentrated on the statistical evaluation of a reference specimen where a defect site is known a priori. This approach enables the quantitative evaluation of the images obtained with several different NDT techniques and/or processed by several different algoinhms, applied to the same test specimen. The basic processing idea is to select both sound and defect areas in each image to be analysed and to characterise a statistical difference between them. The following statistical criteria were used in this study.

I. Signal-to-noise ratio

$$
S N R=\frac{\bar{T}_{d}-\bar{T}_{n d}}{s_{n d}}
$$


where $\bar{T}_{d} \bar{T}_{n d}$ are the mean temperatures in defect and non-defect areas, and $s_{n d}$ is the standard deviation in a non-defect area.

II. Z-criterion:

$$
\hat{z}=\frac{\left|\bar{T}_{d}-\bar{T}_{n d}\right|}{\sqrt{\frac{s_{d}^{2}}{n_{d}}+\frac{s_{n d}^{2}}{n_{n d}}}}
$$

where $n_{n d}$ and $n_{d}$ are the numbers of pixels in the areas to be compared, and $s_{d}$ is the standard deviation in a defect area.

III. D-criterion (Kolmogoroff-Smirnoff's test):

$$
\hat{D}=\max \left|\left(\frac{F_{d}}{n_{d}}-\frac{F_{n d}}{n_{n d}}\right)\right|
$$

where $F_{d}$ and $F_{n d}$ are the two empirical cumulative frequencies divided by the corresponding sample sizes $n_{n d}$ and $n_{d}$ (values $F_{d}$ and $F_{n d}$ are obtained from the statistical frequency distributions).

IV. Probability of false alarm $P_{f . a .}$.

V. Probability of detection $P_{d}$.

The details on the chosen statistical criteria could be found in [6].

The statistical behaviour of the RRT results has been studied by using the ThermoStat computer program developed at Tomsk Polytechnic University for RRT purposes. The program allows the selection of arbitrary areas in images of an arbitrary format in order to calculate corresponding histograms and to determine the statistical criteria.

\subsection{Defect maps}

The simplest defect map is a binary image where a unity pixel value corresponds to a defect area, and zero means a sound background. Defect maps are characterised with certain statistical parameters. The classical decision making discussed in the previous section prompts the following algorithm for making a defect map: $T(i, j)=1$ if $T(i, j) \geq T_{t h r}$, otherwise $T(i, j)=0$ (here $T_{t h r}$ is the decision making threshold). A defect map created by this algorithm will be characterised by particular values of false alarm and correct detection.

\section{The reference specimen and the experimental procedures}

The test specimen was a $2 \mathrm{~mm}$ thick (16-ply) CFRP containing an impact damaged area. The shape and in-depth distribution of the damage were obtained independently by using the ultrasonic C-scan method (see fig. 1a and Section 4.4). On the impacted surface, there were no visible signs of damage, and the point of the very impact was marked with a small white cross (fig. $1 \mathrm{~b}$ ). On the back face, a $10 \mathrm{~mm}$ length crack was clearly visible in the ply direction (fig. 1c).

For the generalisation of the results of all teams participating in the exercise, two main parts of the impact damage were selected for statistical data analysing: Area A and Area $\mathbf{B}$ (fig. 1d). However, single delaminations occurred in the corresponding ply directions. The shallower Area $\mathbf{A}$ has been observed as two adjacent circled zones which ultrasonically proved to consist of details barely visible with $I R$ thermography. The deeper Area B resembling two almonds, completed a pattern consistent with the known "butterfly-like" form of impact damage. Fig. 1d and $1 e$ show how single square areas have been located in the specimen part occupied by the defect. 
http://dx.doi.org/10.21611/qirt.1998.005

The six participants applied up to seven test techniques that differed in both hardware and software. In table 1 these techniques are grouped according to the two fundamentally different forms of the heating procedure.

Table 1. Dynamic TNDT techniques used in the Round Robin test

\begin{tabular}{|c|c|c|}
\hline Technique & Heating parameters & Image recording system \\
\hline \multicolumn{3}{|c|}{ Flash-or square-pulse heating } \\
\hline $\begin{array}{l}\text { Square Pulse } \\
\text { Thermography } \\
\text { (SPT) [7] }\end{array}$ & 3 s $1000 \mathrm{~W}$ flood lamps & $\begin{array}{c}\text { "Thermovision-470" } \\
\Delta T=0.2^{\circ} \mathrm{C}\end{array}$ \\
\hline $\begin{array}{l}\text { Early Detection } \\
\text { Thermography } \\
\text { (EDT) [4] }\end{array}$ & $\begin{array}{c}4 \mathrm{~ms} 0.5 \mathrm{~J} / \mathrm{cm}^{2} \text { flash } \\
\text { tubes }\end{array}$ & $\begin{array}{l}\text { FPA Amber } 4128 \\
\qquad \Delta T=0.007^{\circ} \mathrm{C}\end{array}$ \\
\hline $\begin{array}{l}\text { Square Pulse Phase } \\
\text { Thermography } \\
\text { (SPPT) [8] }\end{array}$ & 2 s $7200 \mathrm{~W}$ flood lamps & $\begin{array}{l}\text { FPA Cincinnati Electronics } \\
\qquad \Delta T=0.025^{\circ} \mathrm{C}\end{array}$ \\
\hline $\begin{array}{l}\text { Flash Pulse Phase } \\
\text { Thermography } \\
\text { (FPPT) [5] }\end{array}$ & $10 \mathrm{~ms} 9 \mathrm{~kJ}$ flash tubes & $\begin{array}{c}\text { "Thermovision-900" LW } \\
\Delta T=0.1^{\circ} \mathrm{C}\end{array}$ \\
\hline $\begin{array}{l}\text { Dynamic Thermal } \\
\text { Tomography } \\
\text { (DTT) [3] }\end{array}$ & $10 \mathrm{~ms} 9 \mathrm{~kJ}$ flash tubes & $\begin{array}{c}\text { "Thermovision-900" LW } \\
\qquad \Delta T=0.1^{\circ} \mathrm{C}\end{array}$ \\
\hline \multicolumn{3}{|c|}{ Thermal wave heating } \\
\hline $\begin{array}{l}\text { Optical Lock-in } \\
\text { Thermography } \\
\text { (OLT) [1] }\end{array}$ & $\begin{array}{c}0.03 \mathrm{~Hz}, 0.06 \mathrm{~Hz} \text { and } \\
0.12 \mathrm{~Hz} \text { thermal waves }\end{array}$ & $\begin{array}{l}\text { "Thermovision-900" LW } \\
\qquad \Delta T=0.1^{\circ} \mathrm{C}\end{array}$ \\
\hline $\begin{array}{l}\text { Ultrasonic Lock-in } \\
\text { Thermography } \\
\text { (ULT) [9] }\end{array}$ & $\begin{array}{c}40 \mathrm{kHz} \text { acoustical waves } \\
\text { modulated with the } 0.23 \\
\mathrm{~Hz} \text { frequency }\end{array}$ & $\begin{array}{c}\text { "Thermovision-900" LW } \\
\Delta T=0.1^{\circ} \mathrm{C}\end{array}$ \\
\hline
\end{tabular}

\section{Results and discussion}

\subsection{Temperature dynamics (flash heating)}

Both the front (impacted face) and the rear surface of the test piece were investigated. The thermograms in fig. 2a-c taken at three observation times show how the front-surface thermal pattern of impact damage evolves in time after a $10 \mathrm{~ms}$ flash. Three particular evolution functions are presented in fig. $2 d$ for a temperature signal which is determined against the adjacent reference point. Two black areas in fig. $2 a$ are due to white-paint marker that absorbs a lower amount of energy. The maximum signal in the Point 1 that probably corresponds to the actual impact point occurs at $200 \mathrm{~ms}$. The Area A that looks like twocoupled circles is seen well at $540 \mathrm{~ms}$ (see Point 2 in fig. 2b) slightly rotating in a ply direction with time. The vast deeper delamination that locates at about $1500 \mu \mathrm{m}$ beneath the surface appears at times greater than 1 second (fig. 2c) and was, surprisingly, colder than the background (Point 3 ) in the experiment (ITEF-CNR). A possible explanation of this phenomenon could be as follows: 1) the RRT specimen changed its properties when "flying" over the world or as a result of inadequate (destructive) treatment, or 2) we deal with signal inversion that could occur due to accelerated cooling rate over a defect area long after a flash.

When images are obtained from the rear surface, it could be anticipated that a thermal pattern of the Area B will overshadow the Area A. This is confirmed with the images in fig. $2 e, f$ where a few details of the almond-shape delamination are seen at the two "early" times. 


\subsection{Statistical analysis}

While using the ThermoStat program, the sound and the defect areas have been selected as shown in fig. 1d,e. The positions of single sub-areas were exactly the same for the images of the same format but differed slightly when passing to another format. By multiple trials, divergences in statistical data that arises due to this have been evaluated at about $10-15 \%$. Therefore the correct comparison of the participating techniques is still questionable, and we have used statistical parameters as rather general indicators. Three statistical parameters adopted for statistically distinguishing between the sound and the defect areas are presented in table 2 . Reviewing the data in table 2 , as well as analysing the histograms obtained in the areas of interest, leads to the following conclusions.

- Pixel value distributions in defect areas were typically wider than those in sound areas, probably due to the bell-shaped temperature distributions over buried defects.

- Applying the rule of thumb [6], we have found that, for images of amplitude and phase, these distributions could appear as normal in both sound and defect areas or reveal significant deviations from normality. Timegrams and phasegrams were rather far from being normal.

- The data in table 2 are grouped in regard to which defect area and which specimen surface has been monitored. It has been impossible to analyse defect Areas A and B altogether because in some images the Area $A$ was "above" the background and the Area B was "under" it (it was especially true in phasegrams and in the FPPT source image (fig. 2c).

- Most of results have been obtained in a front-surface reflection test for the Area $\mathbf{A}$ : Analysis of the Area $\mathbf{B}$ has been thoroughly made in a rear-surface reflection test (the EDT technique).

- In the analysed images, all three adopted statistical parameters exceeded their critical thresholds. This simply means that the selected sound and defect areas were always statistically different.

- In the analysis of the Area $A$, these statistical parameters revealed rather good uniformity in exhibiting the "best" image, i.e. their maximum values were observed in the same group of images obtained with the SPPT and ULT techniques. It is worth noticing that source images at $500-1000 \mathrm{~ms}$ also provide similar detection parameters. The data in table 2 demonstrate that, in accordance with the classical detection theory, SNR values greater than five correspond to $100 \%$ of correct detection. The respective values of the $D$-criterion were close to unity. In all such cases, sound and defect histograms were well separated. Statistical presentation of timegrams has been poor because of their specific characteristics.

- The deeper defect Area B has been noticed on the front surface with only a limited number of techniques. We reiterate that, in the DTT and FPPT experiments, this area was seen a little colder than the background (see the source image in fig. 2c). It is notable that this area is well resolved in the FPPT phasegram having provided up to $94 \%$ of detection (table 2). The use of a relatively long square pulse made this part of the defect undetectable. Also, the EDT has been unable to resolve the Area B on the front surface probably due to lower signals that may appear at shorter times.

- The Area B that located closer to the rear surface has been excellently detected with the EDT method in the rear-surface reflection test. 


\section{http://dx.doi.org/10.21611/qirt.1998.005}

Table 2. The results of statistical data treatment

\begin{tabular}{|c|c|c|c|}
\hline Image & $\hat{D} / D_{c r}$ & SNR & $\begin{array}{c}\text { Pd, \% } \\
(\text { Pf.a }=5 \%)\end{array}$ \\
\hline \multicolumn{4}{|c|}{ Area $A$ : front-surface reflection test } \\
\hline \multicolumn{4}{|l|}{ DTT } \\
\hline Source at $200 \mathrm{~ms}$ & $0.23 / 0.08$ & 0.40 & 10 \\
\hline Source at $540 \mathrm{~ms}$ & $0.88 / 0.08$ & 7.8 & 92 \\
\hline Source at $1610 \mathrm{~ms}$ & $0.98 / 0.08$ & 9.3 & 100 \\
\hline Positive maxigram * & $0.39 / 0.08$ & 0.5 & 10 \\
\hline Normalised source at $540 \mathrm{~ms}^{\text {** }}$ & $0.69 / 0.08$ & 3.6 & 73 \\
\hline Normalised source at $1610 \mathrm{~ms}$ & $0.84 / 0.08$ & 5.6 & 86 \\
\hline \multicolumn{4}{|l|}{ FPPT } \\
\hline Magnitude image at $0.2 \mathrm{~Hz}$ *末 & $0.61 / 0.08$ & 2.6 & 65 \\
\hline Phasegram at $0.2 \mathrm{~Hz}^{\star \star \star \star}$ & $0.93 / 10.07$ & 7.3 & 95 \\
\hline Normalised phasegram at $0.2 \mathrm{~Hz}$ & $0.92 / 0.08$ & 6.5 & 95 \\
\hline \multicolumn{4}{|l|}{ SPT } \\
\hline Source image at $320 \mathrm{~ms}$ & $0.87 / 0.09$ & 3.6 & 91 \\
\hline \multicolumn{4}{|l|}{ EDT } \\
\hline Source image at $630 \mathrm{~ms}$ & $0.96 / 0.13$ & 9.5 & 100 \\
\hline \multicolumn{4}{|l|}{ SPPT } \\
\hline Magnitude image at $0.26 \mathrm{~Hz}$ & $1.00 / 0.09$ & 13.0 & 100 \\
\hline Phasegram at $0.13 \mathrm{~Hz}$ & $1.00 / 0.07$ & 10.0 & 100 \\
\hline Phasegram at $0.39 \mathrm{~Hz}$ & $1.00 / 0.10$ & 6.6 & 100 \\
\hline Phasegram at $3.13 \mathrm{~Hz}$ & $0.93 / 0.12$ & 4.8 & 94 \\
\hline Phasegram at $8.33 \mathrm{~Hz}$ & $0.69 / 0.14$ & 2.1 & 63 \\
\hline \multicolumn{4}{|l|}{ OLT } \\
\hline Phasegram at $0.03 \mathrm{~Hz}$ & $0.95 / 0.08$ & 8.8 & 99 \\
\hline Phasegram at $0.06 \mathrm{~Hz}$ & $0.75 / 0.08$ & 3.2 & 77 \\
\hline Phasegram at $0.12 \mathrm{~Hz}$ & $0.63 / 0.08$ & 5.8 & 64 \\
\hline \multicolumn{4}{|l|}{ ULT } \\
\hline Magnitude image at $0.23 \mathrm{~Hz}$ & $0.96 / 0.1$ & 13.8 & 100 \\
\hline Phasegram at $0.23 \mathrm{~Hz}$ & $0.96 / 0.1$ & 5.0 & 100 \\
\hline \multicolumn{4}{|c|}{ Area B : front-surface reflection test } \\
\hline \multicolumn{4}{|l|}{ DTT } \\
\hline Source at $1610 \mathrm{~ms}$ & $0.82 / 0.08$ & 3.3 & 83 \\
\hline Normalised source at $1610 \mathrm{~ms}$ & $0.74 / 0.08$ & 2.0 & 72 \\
\hline \multicolumn{4}{|l|}{\begin{tabular}{|l} 
FPPT \\
\end{tabular}} \\
\hline Magnitude image at $0.2 \mathrm{~Hz}$ & $0.40 / 0.08$ & 0.8 & 30 \\
\hline Phasegram at $0.2 \mathrm{~Hz}$ & $0.90 / 0.07$ & 4.3 & 94 \\
\hline Normalised phasegram at $0.2 \mathrm{~Hz}$ & $0.83 / 0.07$ & 3.1 & 87 \\
\hline \multicolumn{4}{|l|}{ SPPT } \\
\hline Phasegram at $0.13 \mathrm{~Hz}$ & $0.58 / 0.07$ & 2.1 & 61 \\
\hline \multicolumn{4}{|l|}{ Area B : rear-surface reflection test } \\
\hline \multicolumn{4}{|l|}{ SPT } \\
\hline Source image at $320 \mathrm{~ms}$ & $0.96 / 0.05$ & 7.2 & 100 \\
\hline \multicolumn{4}{|l|}{ EDT } \\
\hline Source image at $360 \mathrm{~ms}$ & $0.99 / 0.07$ & 25.3 & 100 \\
\hline Source image at $630 \mathrm{~ms}$ & $0.99 / 0.07$ & 30.1 & 100 \\
\hline
\end{tabular}

* A maxigram contains maximum temperature signals over a defect even appeared at different times

** A normalised image appears as the result of dividing a source image by the image at the end of heating

*** A magnitude image provides distribution of magnitudes at a particular frequency in the Fourier domain

****A phasegram provides distribution of phases at a particular frequency in the Fourier domain 


\subsection{Morphological analysis}

The images shown in fig. 3 (also in colour) are to demonstrate the versatility of the approaches used. They are not necessarily the best for each particular technique but they do illustrate the techniques features well.

First of all, we have to note the "uniqueness" of the ULT image which reveals different parts of the impact damage in a way different from purely "thermal" techniques (fig. 3a). The SPPT image of Fourier magnitude in fig. $3 b$ provides poor details in the shallower Area $\mathbf{A}$ and shows the rather faint footprint of the deeper Area B. Probably this occurs due to relatively long heating within the SPPT technique (2 s). The next FPPT phasegram (fig. $3 c$ ) being synthesised at about the same frequency exhibits more details throughout the delamination. The only rear surface image shown in fig. $3 d$ ensures excellent appearance of the deepest damage area (that is in fact the shallowest one in respect to the rear surface). It seems that early temperature contrast recording makes a surface defect footprint more sharp but is of a little help in detecting deeper defects (to be correct, we need to say that, in our case, the Area B should partially overshadow the Area $\mathbf{A}$ when performing inspection on the rear surface).

\subsection{Defect characterisation}

Analysing features of defect characterisation and related accuracy has not been the subject of this study (for details, a reader is referred to the published works $[4,7,10]$ ). However, the availability of the alternative ultrasonic depthgram allowed evaluation of TNDT potentials in retrieving the in-depth delamination structure.

The thermal depthgrams have been made within the DTT and EDT techniques. The depthgram in fig. $3 f$ was made by superimposing four single tomograms one of which is shown in fig. 3e. This combined tomogram reveals three parts of the impact damage with depth values quite close to much more detailed ultrasonic depthgram (fig. $3 h$ ). However, rotation of particular delaminations due to changing orientation of plies is well seen. The . depthgram in fig. $3 g$ was obtained by combining front- and rear-surface images within the EDT technique. It reveals three-four specific areas of the defect to compare with up to six in the ultrasonic depthgram. The similarity of these two images in defect shape indicates that the defect image formation processes for ultrasonic and thermal waves are of similar nature. The largest delamination was found between plies 14 and 15, i.e. two plies before the rear surface.

\section{Conclusions}

- In this research, an attempt to compare features of several dynamic thermal NDT techniques on a rather formal basis has been made. The analysed techniques have been different: in the type and duration of the thermal stimulus; in the hardware and the image acquisition systems, as well as in the processing algorithms. The techniques included periodic, flash and square pulse heating with results being treated in the space, time and phase domains. In particular, early detection thermography, lock-in thermography, pulse amplitude and phase thermography and thermal tomography have been used to inspect a carbon fibre composite reference specimen with an impact damage defect. An ultrasonic C-scan depthgram has been available for comparing results.

- The results have been evaluated statistically, heuristically and by using a few characterisation algorithms.

- In the adopted statistical approach, differences between pixel value distributions in the defined defect and non-defect areas has been analysed. The three statistical criteria used (signal-to-noise ratio, $D$-criterion and probability of detection) seemed to be uniform and self-consistent means of verifying how the heating protocols, the types of heating and the processing algorithms influence the statistical 
distinguishability of the impact damage area from the adjacent background. The values of the statistical criteria proved to be much higher than corresponding critical values due to the pixel-based nature of decision making. However, the relative comparison of criterion values could serve as the indicator of techniques efficiency. Statistical data treatment allows the making of defect maps as the binary images with pre-described probabilities of false alarm and correct detection. These images are of special interest for end-users.

- The use of square pulse heating has provided high signals over the defect but reduced ability to resolve details in thermal patterns. Thus, use of flash techniques supplied more details on the impact damage structure. The best spatial resolution has been obtained by using the early detection principle with the resulting depthgram being close to its ultrasonic alternative. In one case, the dynamic thermal NDT was able to find a minor delamination not detected ultrasonically.

- When using a particular NDT technique, time or/and phase treatment of data typically provides better results. However, statistical parameters of decision making, even using a sophisticated processing algorithm, could not exceed much those obtained on conventional source images. Thus, the known statement has been confirmed: if, in an original image, a defect is not seen by an experienced operator, it cannot be detected even if cutting-edge algorithms are applied.

- Ultrasonic stimulation of the specimen produces the thermal pattern different from other techniques that could relate to its volumic nature. Thermal tomograms, unlike all other images, are characterised with a particular pixel value distributions, and classical statistical parameters could be hardly used for their evaluation. Tomograms are recommended to be used in characterising the already detected defects.

- Defect characterisation by using thermal tomography and early detection method have resulted in the appropriate depthgrams that were not much different from their ultrasonic counterpart.

- The RRT approach undertaken could be probably used for evaluating test results obtained with other NDT techniques. However, further research should involve a more formalised protocol in both performing experiments and data processing.

\section{REFERENCES}

[1] WU (D.), SALERNO (A.), SCHÖNBACH (B.), HALIN (H.) and BUSSE (G.) - Phasesensitive modulation thermography and its applications for NDE. Wurzbach (R. N.) and Burleigh (D. D.) eds., Thermosense-XIX, SPIE Proc. 3056, 1997, p.176-183.

[2] AAMODT (L. C.), MACLACHLAN SPICER (J. W.) and MURPHY (J. C.) - Analysis of Characteristic Thermal Transit Times for Time-Resolved Infrared Radiometry Studies of Multilayered Coatings. J. Appl. Phys., 68, 1990, p.6087-6097.

[3] VAVILOV (V.), MALDAGUE (X.), PICARD (J.) et al. - Dynamic thermal tomography: new NDE technique to reconstruct inner solids structure using multiple IR image processing.. Thompson (D. O.) and Chimenti (D. E.) eds., Rev. of Progress in Quant. NDE, vol.11, Plenum Press, New York, 1992, p.425-432.

[4] KRAPEZ (J.-C.) and BALAGEAS (D.) - Early detection of thermal contrast in pulsed stimulated infrared thermography. In: Proc. of the Eurotherm Seminar 42 "Quantitative Infrared Thermography-QIRT'94", Aug.23-26, 1994, Sorrento, Italy, p.260-266.

[5] MALDAGUE (X.) and MARINETTI (S.) - Pulse phase infrared thermography. J. Appl. Phys., 79, 1996, p.2694-2698.

[6] SACHS (L.) - Applied Statistics. A Handbook of Techniques.-Springer-Verlag New York Inc., 1982.-706 p.

[7] ALMOND (D. P.) and LAU (C. K.) Defect sizing by transient thermography. I. An analytical treatment. J. Phys. D: Appl. Phys., 27, 1994, p.1063-1069. 
[8] COUTURIER (J.-P.) and MALDAGUE (X.) - Pulsed phase thermography of aluminum specimens. Wurzbach (R. N.) and Burleigh (D. D.) eds., Thermosense-XIX, SPIE Proc. 3056, 1997, p.170-175.

[9] RANTALA (J.), WU (D.) and BUSSE (G.) - NDT of polymer materials using lock-in thermography with water-coupled ultrasonic excitation. NDE \& E. Intern., 31, 1998, No.1, p.43-49.

[10] VAVILOV (V.), MARINETTI (S.), GRINZATO (E.) and BISON (P.) - Thermal tomography, characterisation and pulse phase thermography of impact damage in CFRP, or why endusers are still reluctant about practical use of transient IR thermography. Snell (J. R.) and Wurzbach (R. N.) eds., Thermosense-XX, SPIE Proc. 3361, 1998, p.275-281.

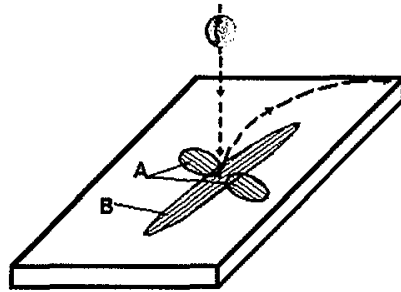

a)

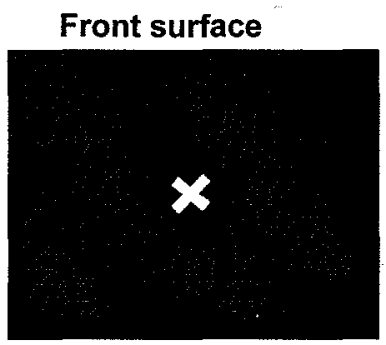

b)

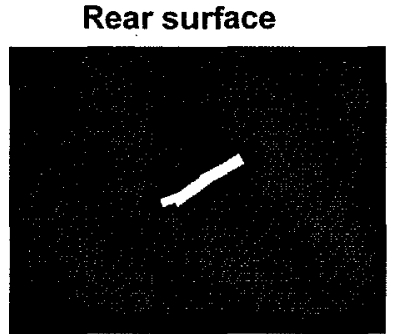

c)
Defect area $A$

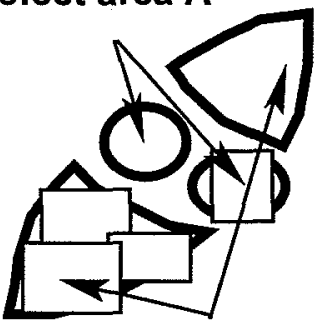

Defect area B

d)

Fig. 1. Defining areas of interest in the inspection of the Round Robin Test CFRP specimen:

a - impacting the specimen,

$b$-front surface with the cross marker,

c - rear surface with the visible crack,

d -locating the defect areas for the statistical analysis,

$e-$ locating the sound area for the statistical analysis. 
http://dx.doi.org/10.21611/qirt.1998.005

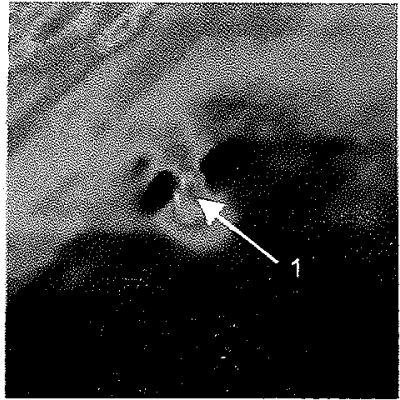

a)

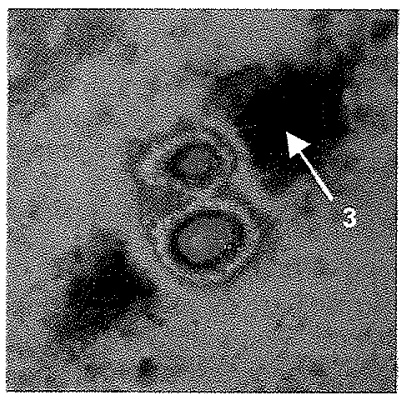

c)

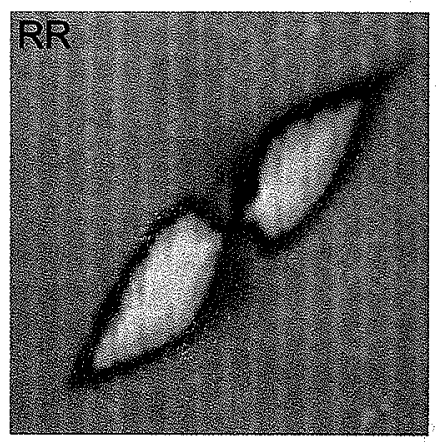

e)

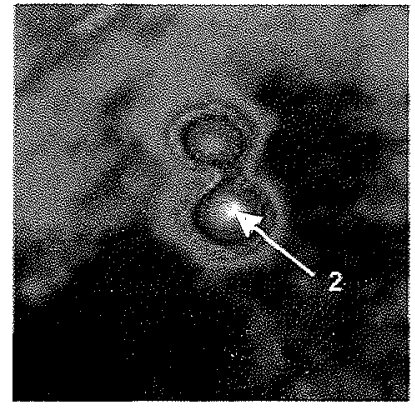

b)

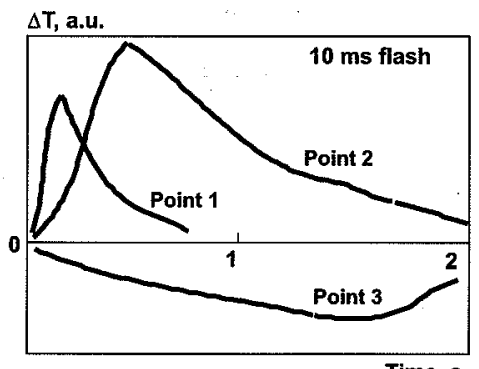

Time, s

d)

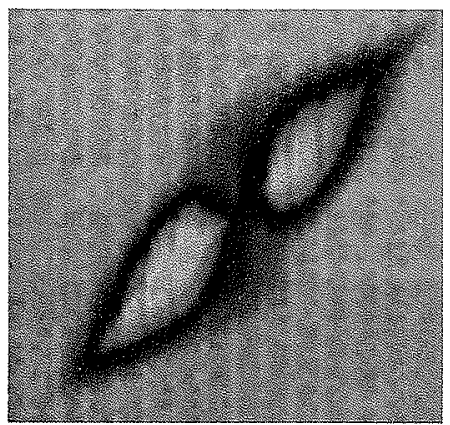

f)

Fig. 2. Image presentation in the flash procedure:

a - ITEF-CNR ( $9 \mathrm{~kJ} 10 \mathrm{~ms}$ flash, THV- 900 , front surface, observation time $200 \mathrm{~ms}$ ),

$\mathrm{b}$ - same as a), $540 \mathrm{~ms}$,

c-same as a), $1600 \mathrm{~ms}$,

$d$-same as a), time evolution of a temperature signal for three points in the defect area,

e - ONERA (0.5 J/cm 4 ms flash, Amber, rear surface, observation time $360 \mathrm{~ms}$ ),

$f$-same as e), $630 \mathrm{~ms}$. 
http://dx.doi.org/10.21611/qirt.1998.005

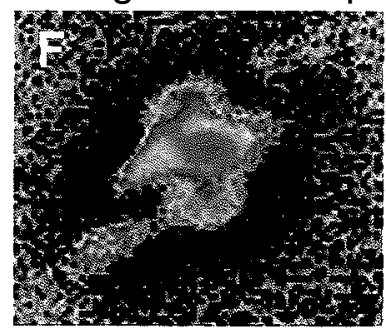

a)

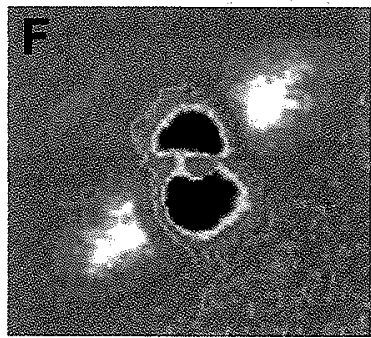

c)

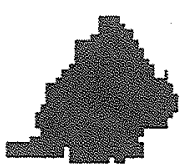

e)

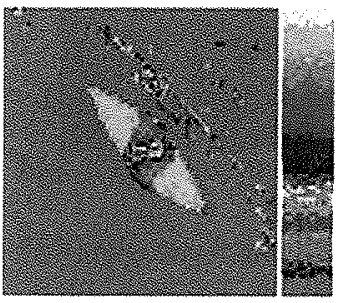

g)

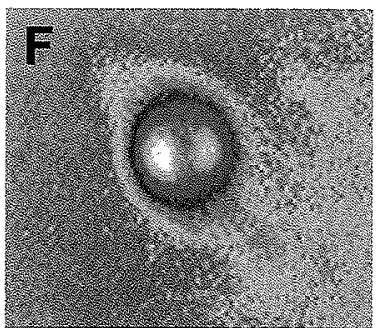

b)

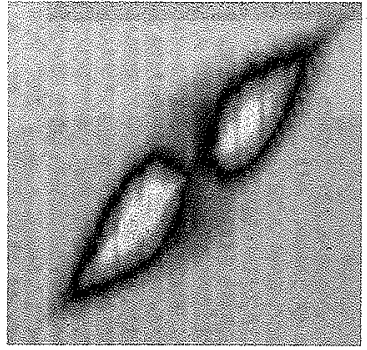

d)

Depth in $\mu \mathrm{m}$

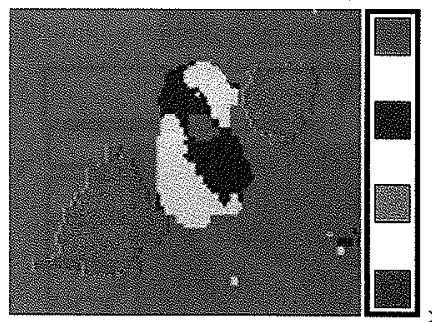

f)

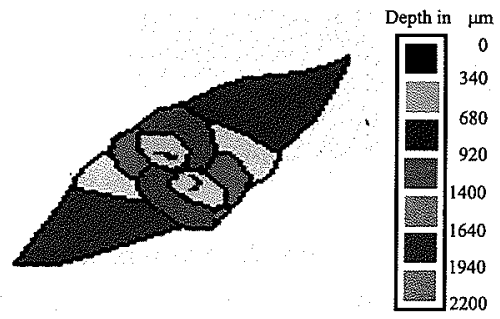

h)

Fig. 3. Image presentation in dynamic thermal NDT of impact damage in the $2 \mathrm{~mm}$ carbon fibre composite specimen ( $F$ - front surface, $R$ - rear surface): a - ULT, phasegram at $0.23 \mathrm{~Hz}$, b - SPPT, magnitude at $0.26 \mathrm{~Hz}$, c - FPPT, phasegram at $0.2 \mathrm{~Hz}, \mathrm{~d}$ - EDT, source image at $630 \mathrm{~ms}$, e - DTT, tomogram of the $0.7-1 \mathrm{~mm}$ layer, f - DTT, combined tomogram (depthgram), g - EDT, depthgram, h - ultrasonic depthgram. 